Apidologie, 1988, 19 (1), 73-84

\title{
ZUR LANGFRISTIGEN VORHERSAGE DER PHYSOKERMES-FICHTENTRACHT
}

\author{
H. PECHHACKER \\ HBLVA für Wein- und Obstbau mit Institut für Bienenkunde, Klosterneuburg ; \\ Abteilung Bienenzüchtung, A-3293 Lunz am See
}

\section{ZUSAMMENFASSUNG}

Durch die Auszählung der Überwinterungslarven (L2) zur Kontrolle der Besatzdichte von Physokermes hemicryphus und durch die Kontrolle der davon überlebenden und honigenden Imagines (I) bzw. des Parasitierungsgrades (PG) und Überlebensrate (UR) dieser Imagines konnte eine langfristige Vorhersagmethode des Befallverlaufes und damit der Lecanientracht gefunden werden. Wenn der Parasitierungsgrad zunimmt bzw. die Überlebensrate der Lecanien abnimmt, nimmt die Besatzdichte dieser Lecanien mit einer Verzögerung von zwei Jahren ebenfalls ab.

Es gibt signifikant gute und schlechtc Wirtsbäume bzw. Standorte. Eine Kontrolle zur gleichen Entwicklungszeit der Lecanien ermöglicht auf Dauer das Auseinanderhalten permanent besserer von permanent schlechteren Wirtsbäumen bzw. Standorten. Standorte mit Waldameisenvorkommen sind bessere Lecanientrachtgebiete.

Die Lecanientracht - verursacht vor allem durch Physokermes hemicryphus Dalm. (Homoptera, Coccidae) - ist in manchen Gebieten Mitteleuropas die wirtschaftlich bedeutendste Tracht für die Imkerei (ARNHART, 1924 ; FosSel, 1960 ; Kloft et al., 1985 ; Pechhacker, 1976 ; Schmutterer, 1965 u.a.).

In früheren Arbeiten wurden Methoden beschrieben, die eine möglichst sichere und zeitgerechte Vorhersage dieser Tracht gewährleisten (PECHHACKER, 1976) bzw. es wurde beschrieben, welchen Einfluß die Wirtspflanze und deren Standort auf die Befallsdichte ausüben (РесннаскеR, 1984, 1985). Fossel (1960) erwähnte ebenfalls Möglichkeiten, die Fichtentracht vorherzusagen.

Die von verschiedenen Autoren (z.B. Maquelin, 1974 ; Müller, 1961 ; SCHeURER, 1967 ; Liebig et al., 1982 u.a.) für die Lachnidentracht beschriebenen Methoden eignen sich kaum für die Vorhersage der Lecanientracht. In der vorliegenden Arbeit wurden folgende Fragen untersucht :

1) Bestehen Unterschiede in der Besatzdichte (Ergiebigkeit der Tracht) zwischen den einzelnen Fichten innerhalb eines Standortes und Jahres bzw. zwischen den verschiedenen Standorten innerhalb der einzelnen Jahre? 


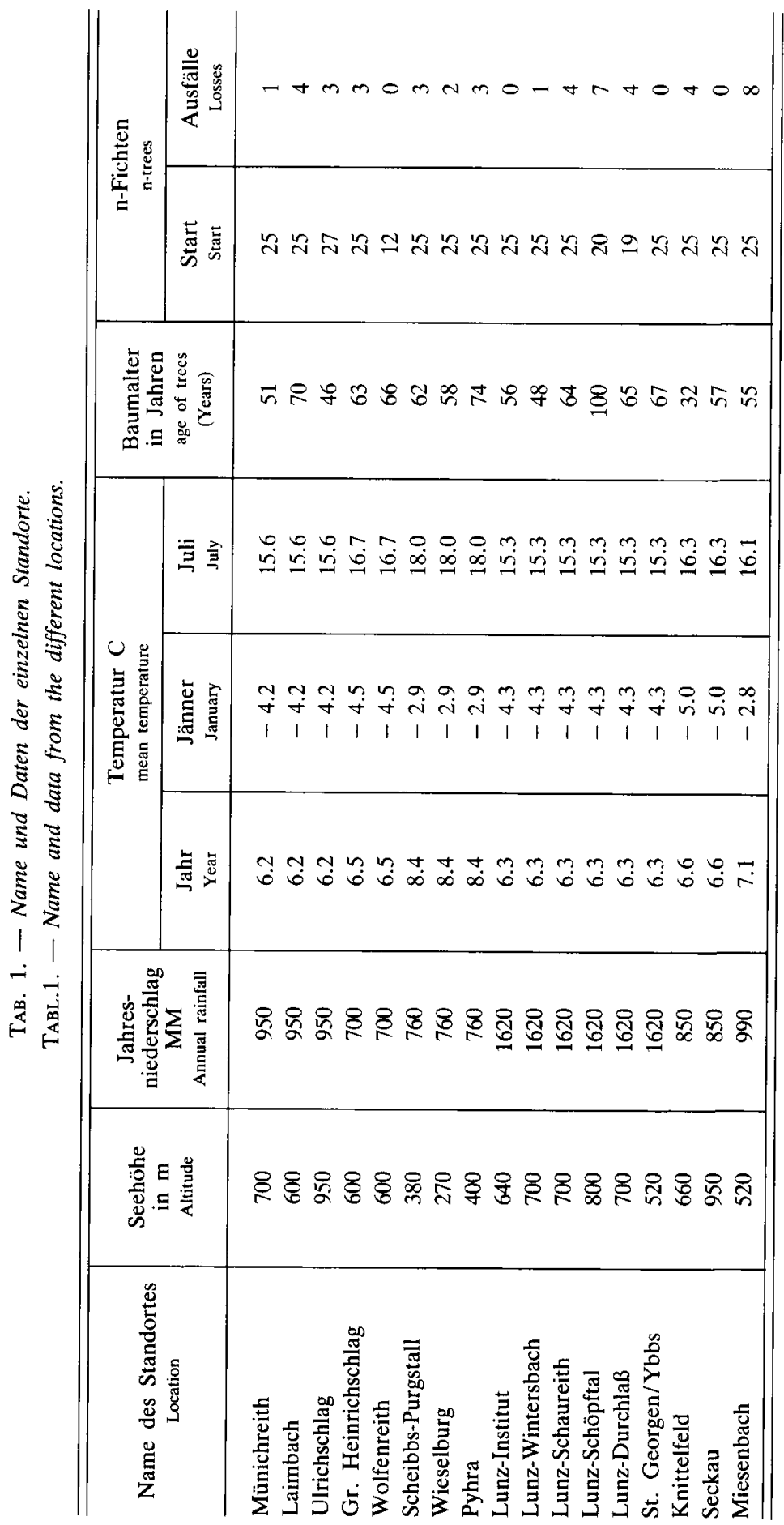


2) Hat die Anzahl der verlorengegangenen Lecanien zwischen der Zählung im Winter (L2) und dem Imaginalstadium (I), (gezählt im Sommer, = « Überlebensrate " der L2 in Prozent) einen Einfluß auf die Populationsentwicklung (= Trachtentwicklung) der Lecanien ? Welche Rolle spielen diesbezüglich die Prädatoren der Physokermes-Arten bzw. die Ameisen ?

3) Ermöglicht die Beantwortung der Fragen 1) und 2) eine langfristige Vorhersage der Physokermes-Fichtentracht?

\section{METHODIK}

Die Populationsschwankungen der Physokermes-Arten wurden aus den Jahren 1975 bis 1984 auf 17 Standorten aus Niederösterreich und der Steiermark (siehe Tab. 1) mit insgesamt 352 Fichten nach der von uns beschriebenen Methode (Pechracker, 1976) festgestellt. Diese Methode wurde insofern verändert, als die L2 nur noch im letzten Triebjahr (das ist der Quirl des Maitriebansatzes des jeweils vorhergegangenen Jahres) jeweils 25 Quirle pro Fichte ausgezählt wurden.

Die Proben wurden von Dezember bis März genommen. Die Auszählung erfolgte im Labor unter einem Stereomikroskop bei zehn- bis fünfzehnfacher Vergrößerung. Zu den Auszählungen der L2 wurden die Zweigproben von den Fichten nur aus dem unteren Kronenbereich (maximal 6-7 m über dem Erdboden) entnommen. Dies ist gerechtfertigt, weil Untersuchungen über die Verteilung der Lecanien im Kronenbereich zeigten, daß nur bei starkem Besatz eine Zunahme des Besatzes pro Quirl im unteren Kronenbereich zu verzeichnen ist. Bei schwachem Besatz ist die Verteilung über den Kronenbereich gleichmäßig.

Nach der gleichen Methode wurden von denselben Fichten zwischen Anfang Juli bis Mitte August die Imagines ausgezählt (jeweils am Maitriebansatz des Vorjahres). Die Überlebensrate in Prozent (ÜR) wurde aus dem Verhältnis der im Sommer gefundenen I zu den auf den jeweiligen Fichten im Winter gezählten L2 errechnet.

Auf den Standorten Laimbach und Ulrichschlag wurde jeweils im August der Prozentsatz der parasitierten oder beraubten Lecanien untersucht. Diese zwei Standorte waren in Bezug auf Klima und Boden vergleichbar. Sämtliche Fichten eines Standortes wurden von Ameisen (Formica sp.) belaufen.

Generell wurden alle Fichten bezüglich des Ameisenbelaufes kontrolliert.

Die statistische Auswertung erfolgte der Datenstruktur entsprechend hauptsächlich mit Hilfe nichtparametrischer Verfahren (SACHS, 1972).

\section{ERGEBNISSE}

Es konnte zwischen den Standorten und Jahren ein deutlicher Unterschied in der durchschnittlichen Besatzdichte festgestellt werden ( $P$ jeweils $<0,01$ ).

Der Durchschnittsbesatz der einzelnen Standorte aus allen Jahren schwankte zwischen 0,06 (Lunz-Wintersbach, niedrigster durchschnittlicher Besatz 1985 mit 0,004 L2 pro Quirl) und 2,09 (Laimbach, höchster durchschnittlicher Besatz 1982 mit 4,97 L2 pro Quirl) L2 pro Quirl. Der Durch- 
schnittsbesatz aller Stände zusammen war 1978 mit 0,97 L2 pro Quirl am höchsten und mit $0,107 \mathrm{~L} 2$ pro Quirl 1980 am niedrigsten.

Auf vier der 17 Standorte wurden die Auszählungen der Populationsdichte und der Überlebensrate bereits seit 1969 durchgeführt. Diese Ergebnisse sind in Abb. 1 dargestellt. Aus dieser Abbildung ist an Hand der Populationskurve der L2 der Abundanzverlauf zu erkennen. Die Populationskurve verlief auf allen Standorten annähernd parallel.

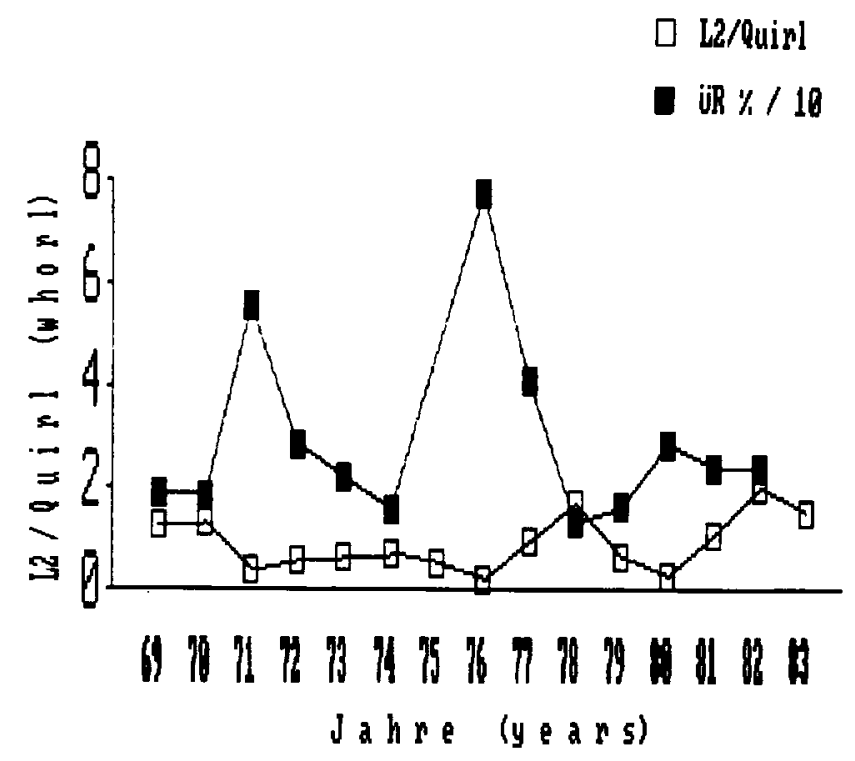

Aвв. 1. - Durchschnittlicher L2-Besatz (leere Vierecke) und Uberlebensrate in Prozent (schwarze Vierecke). Es wurden Durchschnittswerte der Standorte Laimbach, Scheibbs-Purgstall, Lunz und Knittelfeld gebildet.

FIG. 1. - Mean density of overwintering larvae (number of L2 per whorl; open squares) and the survival rate (ÜR) in prozent (solid squares).

Mean values of locations at Laimbach, Scheibbs-Purgstall, Lunz and Knittelfeld.

In Abb. 2 werden die Unterschiede in der Besatzdichte zwischen einem «guten » und einem «schlechten» Standort dargestellt. An Hand der Zählungen zeigten sich auch signifikante Unterschiede in der Besatzdichte zwischen den einzelnen Fichten innerhalb eines Standortes $(P<0,01)$.

Der Durchschnittsbesatz pro Baum und Jahr von allen Standorten betrug $0,59( \pm 1,59) \mathrm{L} 2$ pro Quirl. Die am stärksten besetzte Fichte wies einen Durchschnittsbesatz von $6,89( \pm 5,88)$ L2 pro Quirl und Jahr auf. Die am schwächsten besetzte Fichte hatte in allen Jahren in den jährlich untersuchten 25 Quirlen keinen Besatz (0 L2 pro Quirl). Von dem Standort Laimbach wird die Besatzdichte (L2 pro Quirl) von vier verschieden stark befallenen Fichten 


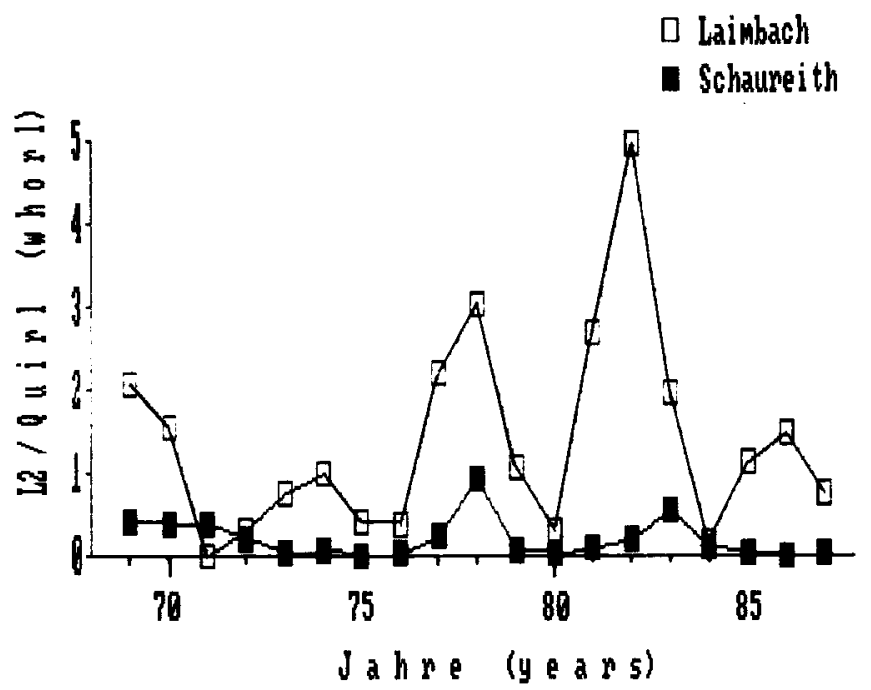

Aв8. 2. - Gegenüberstellung eines « güten" (Laimbach) und eines "schlechten "Standortes (Schaureith).

Fig. 2. - Comparison of a "good" (Laimbach) and a "bad" location (Schaureith).

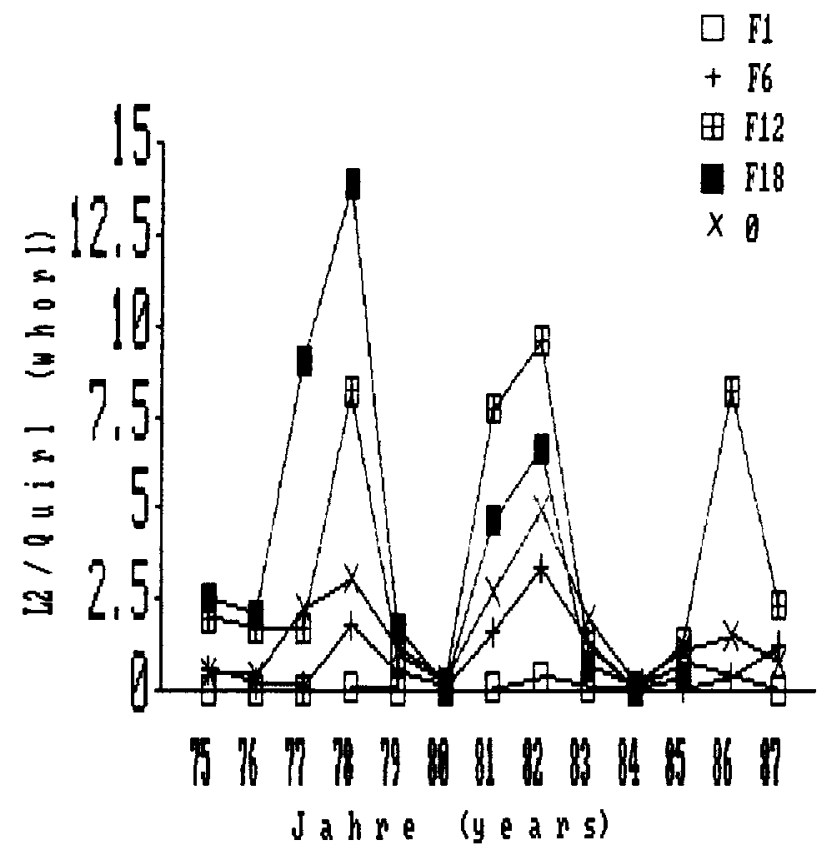

Авв. 3. - Der individuelle Besatzverlauf der Fichten 1, 6, 12 und 18, verglichen mit dem Durchschnittsbesatz ( $\varnothing)$ des Standortes Laimbach 1975-1987.

FIG. 3. - The individual density of $L 2$ of tree numbers $1,6,12$ and 18 compared to the mean density ( $\varnothing$ ) at Laimbach in 1975-1987. 
in der Abb. 3 mit dem Durchschnittsbesatz dieses Standortes verglichen. Es soll in diesem Beispiel der statistisch gesicherte unterschiedliche Befall der einzelnen Fichten anschaulich gemacht werden.

Die Auszählung der Imagines (I) zeigte weitgehend gleichartige Ergebnisse. Der Durchschnittsbesatz bei 327 ausgewerteten Fichten betrug 0,20 $( \pm 0,40)$ Imagines pro Quirl. Von den im Winter ausgezählten Zweitlarven (L2) entwickelten sich in den einzelnen Jahren prozentuell verschieden viele Tiere zu fertigen Imagines (I, Tab. 2). In dieser Tabelle 2 wird die Überlebensrate in Prozent von zwei Standorten (52 Fichten) mit generellem Ameisenbelauf mit der Überlebensrate von acht anderen Standorten ohne Ameisenbelauf (insgesamt 198 Fichten) verglichen. Abb. 1 zeigt die Überlebensrate in Prozent im Vergleich mit dem L2-Besatz zusammengefaßt aus den Jahren 1969-1982. Aus dem Verlauf der Besiedlungsdichte an L2 ist eine zyklische Fluktuation erkennbar. Die Überlebensrate als Ausdruck des Gegenspielerkomplexes verläuft entgegengesetzt zur Kurve der Besiedlungsdichte.

TAB. 2. - Die Überlebensrate $=\ddot{U R}=$ Prozent der Images, bezogen auf die Zahl der $L 2$ der Winterzählung.

TABL.2. - Survivalrate $=\ddot{U} R=$ percent of imagines, referring to the number of L2 counted in winter.

\begin{tabular}{|c|c|c|c|c|c|c|c|c|c|}
\hline \multirow{3}{*}{$\begin{array}{l}\text { Jahr } \\
\text { (year) }\end{array}$} & \multicolumn{3}{|c|}{$\begin{array}{c}\text { Mit Ameisen } \\
\text { (52 Fichten) } \\
\text { with ants } \\
(52 \text { trees })\end{array}$} & \multicolumn{3}{|c|}{$\begin{array}{c}\text { Ohne Ameisen } \\
\text { (198 Fichten) } \\
\text { without ants } \\
(198 \text { trees })\end{array}$} & \multicolumn{3}{|c|}{$\underset{\text { total }}{\text { Gesamt }}$} \\
\hline & \multicolumn{2}{|c|}{$\begin{array}{l}\text { Anzahl der } \\
\text { number of }\end{array}$} & \multirow{2}{*}{$\begin{array}{l}\text { URR } \\
\text { in \% }\end{array}$} & \multicolumn{2}{|c|}{$\begin{array}{c}\text { Anzahl der } \\
\text { number of }\end{array}$} & \multirow{2}{*}{$\begin{array}{l}\text { ÜR } \\
\text { in \% }\end{array}$} & \multicolumn{2}{|c|}{$\begin{array}{l}\text { Anzahl der } \\
\text { number of }\end{array}$} & \multirow{2}{*}{$\begin{array}{l}\text { ÜR } \\
\text { in \% }\end{array}$} \\
\hline & L2 & I & & L2 & I & & $\mathrm{L} 2$ & I & \\
\hline 1976 & 462 & 401 & 86.8 & 614 & 405 & 66.0 & 1076 & 806 & 74.91 \\
\hline 1977 & 1590 & 781 & 49.1 & 3450 & 1426 & 41.3 & 5040 & 2207 & 43.79 \\
\hline 1978 & 3269 & 811 & 24.8 & 5090 & 828 & 16.3 & 8359 & 1639 & 19.61 \\
\hline 1979 & 1657 & 137 & 8.3 & 1924 & 388 & 20.2 & 3581 & 525 & 14.66 \\
\hline 1980 & 226 & 189 & 83.6 & 652 & 321 & 49.2 & 878 & 510 & 58.09 \\
\hline 1981 & 2477 & 446 & 18.0 & 3820 & 750 & 19.6 & 6297 & 1196 & 18.99 \\
\hline 1982 & 1184 & 558 & 47.1 & 5659 & 1380 & 24.4 & 6843 & 1938 & 28.32 \\
\hline $\begin{array}{c}\text { Summe } \\
\text { bzw. } \varnothing \\
\text { total or } \\
\text { mean }\end{array}$ & 10865 & 3323 & 30.58 & 21209 & 5498 & 25.92 & 32074 & 8821 & 27.50 \\
\hline
\end{tabular}

Die Daten von 1969 bis 1974 wurden auf diesen Standorten von noch nicht numerierten Fichten und nach einer etwas anderen Methode erhoben (Pechiacker, 1976). An Hand der Daten von drei vergleichbaren Standorten 
TAB. 3. - Parasitierte oder beraubte Tiere der Gattung Physokermes auf den Standorten

"Ulrichschlag" (= mit Ameisen) und "Laimbach" (= ohne Ameisen). Die Imagines wurden in annähernd gleicher Anzahl für die Untersuchung von den numerierten Fichten beider Standorte entnommen.

TABL. 3. - Parasitized or robbed Physokermes from the places "Ulrichschlag " (= with ants) and "Laimbach" (= without ants). Imagines for this investigation were taken in about the same quantity from the numbered trees of both locations.

\begin{tabular}{|c|c|c|c|c|c|c|c|c|c|}
\hline \multirow{3}{*}{$\begin{array}{l}\text { Jahr } \\
\text { (year) }\end{array}$} & \multicolumn{3}{|c|}{$\begin{array}{l}\text { Mit Ameisen } \\
\text { with ants }\end{array}$} & \multicolumn{3}{|c|}{$\begin{array}{c}\text { Ohne Ameisen } \\
\text { without ants }\end{array}$} & \multicolumn{3}{|c|}{$\begin{array}{c}\text { Gesamt } \\
\text { total }\end{array}$} \\
\hline & \multicolumn{2}{|c|}{$\begin{array}{l}\text { Zahl der Imagines } \\
\text { Number of Imagines }\end{array}$} & \multirow{2}{*}{$\begin{array}{c}\% \\
\text { parasi- } \\
\text { tiert } \\
\text { parasitized }\end{array}$} & \multicolumn{2}{|c|}{$\begin{array}{l}\text { Zahl der Imagines } \\
\text { Number of Imagines }\end{array}$} & \multirow{2}{*}{$\begin{array}{c}\% \\
\text { parasi- } \\
\text { tiert } \\
\text { parasitized }\end{array}$} & \multicolumn{2}{|c|}{$\begin{array}{c}\text { Zahl der Imagines } \\
\text { Number of Imagines }\end{array}$} & \multirow{2}{*}{$\begin{array}{c}\% \\
\text { parasi- } \\
\text { tiert } \\
\text { parasitized }\end{array}$} \\
\hline & $\begin{array}{c}\text { Gesamt } \\
\text { total }\end{array}$ & $\begin{array}{l}\text { parasi- } \\
\text { tiert } \\
\text { parasitized }\end{array}$ & & $\begin{array}{c}\text { Gesamt } \\
\text { total }\end{array}$ & $\begin{array}{l}\text { parasi- } \\
\text { tiert } \\
\text { parasitized }\end{array}$ & & $\underset{\text { total }}{\text { Gesamt }}$ & $\begin{array}{c}\text { parasi- } \\
\text { tiert } \\
\text { parasitized }\end{array}$ & \\
\hline 1975 & 429 & 136 & 31.7 & 250 & 169 & 67.6 & 679 & 305 & 44.92 \\
\hline 1976 & 1250 & 606 & 48.5 & 303 & 206 & 68.0 & 1553 & 812 & 52.29 \\
\hline 1977 & 578 & 269 & 46.5 & 720 & 585 & 81.3 & 1298 & 854 & 65.79 \\
\hline 1978 & 128 & 97 & 75.8 & 339 & 304 & 89.7 & 467 & 401 & 85.87 \\
\hline 1979 & 123 & 74 & 60.2 & 312 & 170 & 54.5 & 435 & 244 & 56.09 \\
\hline 1980 & 891 & 384 & 43.1 & 741 & 361 & 48.7 & 1632 & 745 & 45.65 \\
\hline 1981 & 1210 & 560 & 46.3 & 1213 & 455 & 37.5 & 2423 & 1015 & 41.89 \\
\hline 1982 & 398 & 336 & 84.4 & 666 & 453 & 68.0 & 1064 & 789 & 74.15 \\
\hline $\begin{array}{c}\text { Summe } \\
\text { bzw. } \varnothing \\
\text { total or } \\
\text { mean }\end{array}$ & 5007 & 2462 & 49.17 & 4544 & 2703 & 59.49 & 9551 & 5165 & 54.08 \\
\hline
\end{tabular}

(Laimbach, Münichreith und Ulrichschlag) wurden der Einfluß von Besatzdichte, Ameisenbelauf, Jahr und der jeweiligen Wechselwirkung auf die Überlebensrate untersucht.

Nur das Jahr $(\mathrm{P}<0,01)$ und die Wechselwirkung von Jahr $\times$ Besatzdichte $(\mathrm{P}<0,05)$ zeigten einen signifikanten Einfluß. Der Ameisenbelauf zeigte keinen signifikanten Einfluß auf die Überlebensrate $(P>0,1)$. Die in Tab. 2 dargestellten Werte aus den Jahren 1976-1982 zeigen aber, daß die Überlebensrate im Ameisengebiet um durchschnittlich 4,66 Prozent höher ist als ohne Ameisen. Die Ergebnisse über die Auszählung des Parasitierungsgrades auf den vergleichbaren Standorten mit und ohne Ameisen sind in Tab. 3 dargestellt. Die jeweiligen Prädatoren wurden nicht auseinander gehalten. Auffallend war, daß mit Ausnahme eines Jahres der räubernde Schildlausrüßler Anthribus nebulosus FORST. nur selten gefunden wurde. Verschiedene Zehrwespenarten waren mit Sicherheit die Hauptgegenspieler. Unterschiede in der Besatzdichte zwischen dem Standort (unter Einbeziehung aller Standorte) mit oder ohne Ameisen sind signifikant $(P<0,01)$. Auf drei Standorten wurde auch der Prozentsatz (bezogen auf die im Winter gezählten L2) der insgesamt 
überlebenden - d.h. der voll entwickelten, nicht parasitierten und daher reproduktiven - Imagines errechnet. Die Korrelation nach SPEARMAN zwischen den gesamt überlebenden Imagines (TÜ) und dem L2-Besatz des Folgejahres ist mit rs $=0,60$ signifikant $(P<0,05)$. Die partiellen Beziehungen zwischen Besatzdichte an Lecanien und Ameisenbelauf sind signifikant $(P<0,05)$, außer es wurden Seehöhe und Bodenuntergrund gleichzeitig konstant gehalten.

\section{DISKUSSION}

Wie schon in anderen Arbeiten (PechHACKer, 1976 und 1977) dargestellt, scheint die Witterung keinen wesentlichen Einfluß auf die Fluktuation der Physokermes-Arten auszuüben. Dies deckt sich auch mit den Untersuchungen von LIEBIG et al. (1982) an Cinara pectinatae NÖRDL. bzw. den Ergebnissen von Негмвасн (1984) bei Untersuchungen über zwei Zierlausarten auf Linde und Eiche. Die festgestellte signifikant verschiedene Befallsbereitschaft der einzelnen Fichten auch innerhalb eines Standortes besagt, daß es « gute » und «schlechte» Wirtsbäume gibt. Die möglichen Ursachen der standort- und wirtsbaumbedingten verschiedenen Befallsdichte wurde in einer anderen Arbeit besprochen (PECHHACKER, 1985).

Die aus Abb. 1 erkennbare vier- bis fünfjährige zyklische Fluktuation ist offenbar dichteabhängig und wird nach den vorliegenden Untersuchungen wahrscheinlich im wesentlichen von den Gegenspielern gesteuert. Diese Behauptung wird durch die Ergebnisse bezüglich Überlebensrate und Parasitierungsgrad bestätigt (siehe Tab. 2 und 3 bzw. Abb. 1).

Aus den vorliegenden Ergebnissen können eine Reihe bienenwirtschaftlich interessanter Möglichkeiten zur Vorhersage und besseren Ausnutzung der Fichtentracht abgeleitet werden. Wenn mehr reproduktive Lecanien (bezogen auf die im Winter gezählten L2) überleben, steigt die Besatzdichte im Folgejahr an. Sinkt dieser Prozentsatz aber, ist mit einem Rückgang der Populationsdichte und der Tracht zu rechnen. Es ist nicht mehr notwendig, bei jedem in Frage kommenden Standort einer Region (z.B. Nordalpenbereich und nördliches Alpenvorland oder südliches Waldviertel) den L2-Besatz auszuzählen. Es wird für die Praxis genügen, nur auf einem Referenz-Standort einer Region diese Zählungen durchzuführen. Dies erfordert einen wesentlich geringeren Arbeitsaufwand und ermöglicht die gleiche Genauigkeit wie die bisherigen Methoden. Die Ergebnisse zeigten nämlich, daß die Schwankungen der Besiedlungsdichte für größere Regionen gleichartig verlaufen.

Die Fichten der Ameisengebiete bzw. einzelne von Ameisen belaufene Fichten haben in der Regel einen höheren Lecanien-Besatz und liefern daher 
auch eine bessere Honigtautracht (PechHacker, 1976). Die Ansicht, daß Ameisengebiete bessere Trachtgebiete sind, galt bisher nur für bestimmte Lachnidentrachten (Fossel, 1972 ; Kloft et al., 1985 ; Wellenstein, 1958 ; RutTNeR H. und PechнаскеR, 1969 u.a.). Es stellt sich hier allerdings die Frage, ob ein bestimmter Baum deswegen stärker von Lecanien besiedelt wird, weil dieser Baum von Ameisen belaufen wird oder belaufen die Ameisen den Baum, weil er von mehr Lecanien (bzw. Lachniden) besiedelt wird. Diese Frage ist insofern berechtigt, weil der Einfluß der Ameisen auf die Überlebensrate bzw. den Parasitierungsgrad statistisch zwar z.T. abzusichern ist, aber nicht sehr hoch zu sein scheint (siehe Tab. 2 und 3).

Nach den vorliegenden Ergebnissen ist es sehr einfach, die «guten» Standorte oder Fichten von den «schlechten » zu unterscheiden. Man muß zur gleichen Zeit (in Bezug auf den Entwicklungsstand der Lecanien innerhalb eines Jahres) auf dem gewünschten Standort einer Region eine Besatzkontrolle mit bloßen Augen oder einer Taschenlupe durchführen. Die durchschnittliche Anzahl der gefundenen Lecanien gleichen Alters pro Quirl der einzelnen Standorte kann dann verglichen werden. Das ergibt eine Aussage über die zu erwartende Trachtergiebigkeit der einzelnen Standorte. Die positiven Beziehungen zwischen Lecanienbesatz pro Quirl und dem Honigertrag wurden 1976 beschrieben (PechHAcker, 1976).

Eingegangen im Mai 1987. Angenommen im Dezember 1987.

\author{
RÉSUMÉ \\ PRÉVISION A LONG TERME DE LA MIELLÉE D'ÉPICÉA \\ PRODUITE PAR LA COCHENILLE PHYSOKERMES (HOMOPTERA, COCCIDAE)
}

En 17 lieux différents, de 1976 à 1984 (86), sur 352 épicéas (Picea excelsa L.) marqués, on a déterminé la densité de cochenilles en hiver (larves $=\mathrm{L} 2$ ) et en été (imagos $=\mathrm{I}$ ), le nombre de fourmis et, à deux endroits, le taux de parasitisme (Tabl. 1). On a calculé, en pourcentages par rapport aux L2 comptées, le taux de survie et le taux de cochenilles reproductrices non infestées.

Il y a une différence significative de densité de cochenilles par verticille entre les diverses années en un même lieu et entre les divers lieux la même année (Fig. 1). La différence est également significative entre les divers épicéas d'un même lieu, la même année (Fig. 3).

La corrélation entre le taux de survie ou le taux des cochenilles reproductrices non parasitées survivantes d'une part et la densité de population ultérieure d'autre part est également significative (Fig. 1, Tabl. 2 et 3). Les épicéas à forte population de fourmis ont une densité de cochenilles significativement plus élevée.

On peut prédire la miellée d'épicéa provenant de Physokermes de différentes façons :

1) En comparant le nombre de vieilles écailles de Physokermes restant sur les branches ou le nombre d'individus vivants avec les écailles ou les cochenilles du même âge, on peut distinguer de «bons» et de « mauvais» arbres et prévoir ainsi des secteurs de miellée. 
2) L'évolution du taux de survie donne des indications sur la densité de population de Physokermes à laquelle on peut s'attendre et, par là même, sur la miellée de la saison suivante.

Les endroits où les populations de fourmis sont élevées sont généralement de bonnes régions pour la miellée d'épicéa provenant de Physokermes.

\section{SUMMARY \\ LONG-TERM FORECAST OF PHYSOKERMES (HOMOPTERA, COCCIDAE) HONEYDEW FLOW ON SPRUCE}

In 17 different locations, between 1976 and 1984 (86), the density of overwintering larvae (L2), the adult female and the ant visitation of Physokermes sp. were counted on 352 numbered spruce trees (Picea excelsa L.) (Table 1). In two different locations the degree of parasitic infestation was also noticed. Related to the number of counted L2 the survival rate and not infested reproductive Physokermes was calculated (in percent of the number of L2).

A significant difference was observed in the number of Physokermes per whorl between the years in a certain location, and between locations in the same year (Fig. 1). Significant differences in the number of Physokermes were also found between spruce trees in locations and years (Fig. 3).

The correlation between the survival rate, resp. the survival rate plus not infested reproductive Physokermes, and the subsequent population density is significant (Fig. 1, Tabl. 2 and 3). A forecast of the Physokermes honeydew flow can be made in various ways :

1) By comparing the number of old Physokermes scales remaining on the branches or the number of living individuals with scales or Physokermes of the same age, permanently "good " and «bad" trees and areas can be distinguished.

2) The development of the survival rate gives information on the number of Physokermes and the honey flow expected for the following season.

Locations with a higher ant population are better areas for honeydew production also as far as Physokermes is concerned.

\section{LITERATUR}

Arnhart L., 1924. - Der Fichtenhonig. Bienenvater, 56, 19-22.

Fossel A., 1960. - Die Fichtentracht. Bienenvater, 81, 204-229.

Fossec A., 1972. - Die Populationsdichte einiger Honigtauerzeuger und ihre Abhängigkeit von der Betreuung durch Ameisen. Waldhygiene, 9, 185-191.

Heimbach U., 1984. - Freilanduntersuchungen zur Honigtauausscheidung und Populationsdynamik zweier Zierlausarten (Aphidina) an Linde und Eiche im Hinblick auf die Honigtaunutzung durch Honigbienen. Diss. Universität Hannover 1984.

Kloft W., Kunkel H., 1985. - Waldtracht und Waldhonig in der Imkerei. Ehrenwirth-Verlag, München.

Liebig G. und SchlipF U. und Düwel W., 1982. - Witterungsverlauf und Massenwechsel der Grünen Tannenhoniglaus Cinara pectinatae (Nördl.) (Homoptera, Lachnidae) in den Jahren 1977-1981. Apidologie, 13 (3), 275-295. 
Liebig G., SCHLiPf U., 1982. - Die Tannentracht 1981 : Vorhersage und Verlauf. Alig. disch. Imkerztg., 16, 201-204.

Maquelın C., 1974. - Observations sur la biologie et l'écologie d'un puceron utile à l'apiculture. Thèse Eth Zürich.

Müller H., 1961. - Vorschau und Erkundung von Waldtracht. Symp. Genet. Biol. Ital, XI, 85-103.

Pechiacker H., 1976. - Zur Vorhersage der Honigtautracht von Physokermes hemicryphus Dalm. (Homoptera, Coccidae) auf der Fichte (Picea excelsa). Apidologie, 7, 209-236.

Pechiacker H., 1977. - Über die Auswirkung von Umwelteinflüssen auf die Populationsentwicklung der Physokermes-Arten. Apidologie, 8, 451-457.

PechHAcker H., 1984. - Zur Populationsentwicklung der Physokermes-Arten. Diss. Universität für Bodenkultur, Wien.

Pechhacker H., 1985. - Über den Einfluß des Standortes und der Eigenschaften der Wirtspflanze Fichte auf die Befallsdichte der Fichte durch Physokermes sp. Mitt. Klosterneuburg, 85, 218-224.

Ruttner H. und Pechiacker H., 1969. - Die Weißtannentracht 1968. Z. Bienenforsch., 9 (10), 421433.

SACHS L., 1969. - Statistische Auswertungsmethoden. Springer-Verlag, Bcrlin.

SCHeurer St., 1967. - Wir starten den crsten Versuch der Waldtrachtprognose. Garten und Kleintierzucht, C, 6 (11).

SCHMUtTerer H., 1965. - Zur Ökologie und wirtschaftlichen Bedeutung der Physokermes-Arten (Homoptera, Coccidae) an Fichte in Süddeutschland. Z. ang. Entomol., 56, 300-325.

Wellenstein G., 1958. - Die Trophobiose der Waldmeisen und ihre bienenwirtschaftliche Bedeutung. 14. Verh. Ber. dtsch. Ges. ang. Entomol., 109-114. 